\title{
3 Parity Without Imprecise Equality
}

\author{
Chrisoula Andreou
}

In choosing between two options, one might, if time permits, compare the options and attempt to determine if one option is better and the other worse, or if the options are equally good (overall and relative to what matters in the situation at issue, which can include the fit between the options and the taste or preferences of the choosing agent). According to the trichotomy thesis, "the conceptual space of comparability between two [options] is spanned by the trichotomy of relations 'better than,' 'worse than,' and 'equally good': if none of those relations holds, the [options] are incomparable" (in terms of their overall value relative to what matters in the situation at issue). ${ }^{1}$ The trichotomy thesis has been challenged based on the idea that two options that are not rankable as one better than the other or as exactly equally good might, at least intuitively, be positively related to one another as "on a par" (rather than just negatively related to one another as not exactly equally good and not one better than the other). But what is it for two items to be on a par?

A prominent and seemingly promising approach to elucidating parity is to build on the notion of rough equality - understood as involving closeness in value (more on this later) - and to cast the dispute between proponents and opponents of the trichotomy thesis as follows: according to proponents of the trichotomy thesis, all pairs of options that are roughly equally good are, if not exactly equally good, then such that one option is strictly better than the other, though not by much. According to opponents of the trichotomy thesis, this is not so; rather, in some cases, two roughly equally good options are imprecisely equally good - though close in value, they are not rankable in relation to one another ${ }^{2}-$ and it is the relation between such options that the term "on a par" is best reserved for. This suggestion fits quite neatly with the familiar idea, which I will take on board, that if two options are on a par, then, even though the options are not rankable in relation to one another, they are in the same neighborhood in terms of their overall value (i.e., in terms of how 
valuable they are overall) relative to what matters in the situation. ${ }^{3}$ Given this idea, which captures the key features of the notion of parity that I am here concerned with, it is natural to conclude that options that are on a par must be close in value, and so roughly equally good. ${ }^{4}$ But, as I will argue, this is not the case. ${ }^{5}$

Developing a view that I present, in part, elsewhere, ${ }^{6} \mathrm{I}$ will begin by focusing on the construal of parity as imprecise equality and on the case to be made for the possibility of imprecise equality. I think that the case is strong. But, building on the understanding of imprecise equality that I develop here and in some examples and ideas in some of my prior work, I explore the possibility that there can be cases of comparability beyond those acknowledged by the trichotomy thesis that are not cases of imprecise equality but are still cases of parity (wherein the options are not rankable in relation to one another but are in the same neighborhood). I then focus on the question of how two options could be in the same neighborhood in terms of their overall value (or, more precisely, in terms of how valuable they are overall relative to what matters in the situation at hand) if the options are not roughly equally good (and so are not imprecisely equally good). In responding to the question, I explain that, in evaluating a particular group of alternatives, some of which are not rankable in relation to others, one may need to use a "grading system" with wide-ranging evaluative classes. Significantly, when two options fall in the same wide-ranging evaluative class, they need not have the closeness in value necessary to qualify them as roughly equally good. Nevertheless, for options in the same class that, by hypothesis, cannot be ranked in relation to one another and cannot be "graded" using a grading system that employs narrower evaluative classes, counting the options as in the same neighborhood and, relatedly, as on a par seems perfectly appropriate even if the options cannot also be described as roughly equally good.

\section{2}

Items can be compared in all sorts of ways. Books can be compared based on, for example, their innovativeness or, alternatively, on their suitability to keep a fire going. The items of interest in this chapter are options that agents might have to choose between. Moreover, for two options to be comparable in the sense of interest here, it does not suffice that they are comparable in any old way.

For example, two submissions for a creative writing contest are not necessarily comparable in the relevant sense just because one is better relative to one of the factors that matters given the context of choice, such as, say, originality. Similarly, the two submissions are not necessarily comparable in the relevant sense just because one is better in 
terms of a factor that is altogether irrelevant given the choice situation, such as, say, suitability for use as a door stop.

(Andreou 2020a)

Furthermore, as suggested in section 1, the truth of a negative comparative claim such as, for example, that $\mathrm{X}$ and $\mathrm{Y}$ are not equally good, does not suffice to make the options comparable in the sense of interest here. For comparability in the relevant sense to obtain, there must be some positive relation between the options that captures how they compare in terms of their overall value, relative to what matters in the situation at issue, from the point of view of practical reason. Notably, whether there is a positive relation that captures how two options compare in the relevant sense does not depend on whether the two options are themselves good or bad options. In particular, two bad options can be positively related to one another as exactly equally bad.

According to the standard view, all options (in a particular choice situation, given a particular choosing agent) are comparable (in the relevant sense); more specifically, according to the standard view, for every pair of options, one option is either better than the other or the options are exactly equally good. The standard view can be resisted in two ways. First, it might be argued that options are not invariably comparable sometimes there is no positive relation between the options that captures how they compare in terms of their overall value. This is the incomparability thesis. Second (and additionally or else alternatively), it might be argued that comparable options are not always comparable as one better than the other or as exactly equally good. This way of resisting the standard view rejects the aforementioned trichotomy thesis, according to which, if two options are comparable, one option is better than the other, worse than the other, or exactly as good as the other.

Before Ruth Chang's now prominent discussions of the trichotomy thesis, arguments that could be developed as either for the incomparability thesis or against the trichotomy thesis were sometimes automatically cast as arguments for the incomparability thesis. Consider the smallimprovement argument, which originally played the role of the most promising argument for the incomparability thesis. ${ }^{7}$ According to the small-improvement argument, there are options $\mathrm{X}, \mathrm{Y}$, and $\mathrm{X}+-$ where $\mathrm{X}+$ is a slightly improved variation of $\mathrm{X}-$ such that:

(1) $X$ is not better than $Y$

(2) $Y$ is not better than $X$

(3) $X+$ is better than $X$

(4) $X+$ is not better than $Y$

If it is granted (in accordance with the next step in the argument) that if two options are exactly equally good, then anything that is better than 
one of the options will also be better than the other option, it follows from 3 and 4 that $\mathrm{X}$ and $\mathrm{Y}$ are not exactly equally good. But then, given 1 and 2, it follows that $\mathrm{X}$ and $\mathrm{Y}$ are neither one better than the other, nor exactly equally good. ${ }^{8}$

Filling in the small-improvement argument requires providing plausible candidates for $\mathrm{X}$ and $\mathrm{Y}$ (i.e., candidates for which 1-4 all seem clearly true). Prominent proposed cases include cases in which it seems plausible to suppose that the agent knows all there is to know about what matters in the case at hand, such as, for instance, Chang's (2002) coffee and tea case in which, by hypothesis, all that matters is whether and, if so, which drink tastes better to the agent. In Chang's case, the agent "rationally judge[s] that [a particular] cup of Sumatra Gold tastes neither better nor worse [to her] than [a particular] cup of Pearl Jasmine and that although a slightly more fragrant Jasmine would taste better than the original, the more fragrant Jasmine would not taste better than the cup of coffee" (2002: 669). If $X=$ opting for the Pearl Jasmine, $Y=$ opting for the Sumatra Gold, and $\mathrm{X}_{+}=$opting for a slightly more fragrant Jasmine, we have, it seems, the sort of case we need.

But, even if this is right, we can, as Chang does, resist the incomparability thesis. For, it may be that cases that fit the small-improvement argument are best understood as cases that count against the trichotomy thesis, not against the comparability of the options under consideration. And, indeed, this seems like the best way of understanding the challenge raised by Chang's coffee and tea case. Even opponents of the standard view should, it seems, agree that Chang's coffee and tea case is not a case of incomparable options. The options seem roughly equally good. The small-improvement argument suggests that they are not also one better than the other or else exactly equally good, which in turn suggests that they are imprecisely equally good.

The preceding reasoning assumes, following Chang, that the debate between the proponents and opponents of the trichotomy thesis can be fruitfully construed as not a mere terminological dispute but as a substantive dispute about our "ordinary" or "intuitive notions of comparability and incomparability" (Chang 2016: 191-192).

In what follows, I will accept the small-improvement argument as an argument against the trichotomy thesis and consider whether all cases of parity (wherein the options are not rankable in relation to one another but are in the same neighborhood) must be understood as cases of imprecise equality. After saying more about imprecise equality, I will, building on my construal of imprecise equality and on my prior work on parity, provide considerations in favor of the conclusion that the answer is "no." As part of supporting this conclusion, I will focus on explaining how two options can be in the same neighborhood (in terms of how valuable they are overall relative to what matters in the situation) without necessarily being close in the sense implied by rough equality. Note 
that, henceforth, the parenthetical remark in the preceding sentence will often be left implicit, as will my previously repeated remark regarding the notion of parity at issue in this chapter, according to which options that are on a par are not rankable in relation to one another but are in the same neighborhood.

As suggested previously, to say that two options are imprecisely equally good is to say that they are roughly equally good and that they are not also one better than the other or else exactly equally good. But what is it for two options, say X and Y, to be roughly equally good? One thing that seems necessary is that their overall values are close; and for this to hold it must be that were another option, W, considerably (i.e., quite a lot as opposed to just a little) better than one of the original options, it would also be better than the other. Importantly, this necessary condition leaves open the question of whether $\mathrm{X}$ and $\mathrm{Y}$ are precisely comparable to one another. Relatedly, it is compatible with there being an option that is a little better than one of the options but not better than the other; were this ruled out, rough equality would not be compatible with imprecise equality. ${ }^{9}$

Return to Chang's coffee and tea case. Chang's cup of Sumatra Gold and cup of Pearl Jasmine seem roughly equally good; the small-improvement argument suggests that they are not also one better than the other or else exactly equally good; as such, they seem to qualify as imprecisely equally good. Insofar as they are imprecisely equally good, there is some small improvement, $s$, such that improving one of the options with $s$ does not make the improved option strictly better than the alternative. In particular, the small improvement of making the Pearl Jasmine slightly more fragrant does not make it strictly better than the Sumatra Gold. Still, insofar as the Pearl Jasmine and the Sumatra Gold are roughly equally good, it seems like an option that is quite a lot better than one of the options will be better than the other option too; for, if an option that is quite a lot better than one of the options is not better than the other, the original options must not have been close in value. If, for example, a glass of Dom Pérignon is quite a lot better than the cup of Pearl Jasmine but not better than the cup of Sumatra Gold, it seems false to say that the cup of Pearl Jasmine and the cup of Sumatra Gold are roughly equally good.

\section{4}

Now consider the question of whether all cases of parity must be cases of rough equality and, more precisely, cases of imprecise equality. (Note that settling this question does not necessarily settle the question of whether there are any cases of incomparability, which I here leave aside.) Given 
the (partial) description of rough equality provided earlier - according to which "X is roughly equal to $Y$ " implies that, were another option, W, considerably better than one of the options, it would be better than the other option too - there could, I contend, be cases of parity that are not cases of rough equality and so not cases of imprecise equality. In particular, there could be cases in which the options are intuitively characterizable as on a par, and yet there is another option that is quite a lot better than one of the options but not better than the other.

Consider the following case:

Suppose that one is both compassionately and philosophically inclined, and let A [and] B... be defined as follows:

$\mathrm{A}=$ dedicating the summer to compassionately engaging with others by volunteering at a homeless shelter

$\mathrm{B}=$ dedicating the summer to promoting one's philosophical development by completing a book manuscript $\cdots$

Taking into account the context of choice, including any relevant features of the choosing agent, suppose that (from the point of view of practical reason), $A$ is not better than $B$ and $B$ is not better than A. ${ }^{10}$

Now consider an improved version of the compassionate option, call it $\mathrm{A}^{*}$, in which one also provides a temporary foster home for a needy child. A* does not seem roughly as good as A, but quite a lot better. Still, like $\mathrm{A}$ and $\mathrm{B}, \mathrm{A}^{*}$ and $\mathrm{B}$ are so different, it seems like they too might be such that neither is better than the other (from the point of view of practical reason). But then we have, in $\mathrm{A}^{*}$, an option that is quite a lot better than A but not better than B. This suggests that even though A and B seem like they are on a par, they are not imprecisely equally good.

Importantly, this case is not meant to provide decisive support for the view that there can be options that are on a par but not imprecisely equally good. However, once it is supplemented with the reasoning that I will provide for the view that we can make sense of two options being in the same neighborhood without being close in the way that options that are roughly equally good are, it will, I hope, be clear that we should not assume that all cases of parity are cases of imprecise equality. ${ }^{11}$

Interestingly, Chang provides a characterization of options that are on a par that allows for the possibility of options that are on a par but not necessarily imprecisely equally good. In working up to this characterization, Chang (2016: 194) suggests that

we understand value relations in terms of evaluative differences between items [with respect to what matters in the choice situation at issue, where] evaluative differences can be individuated along 
two dimensions: (1) bias or direction, that is, whether the difference favours an option or "points to" one of them, and (2) magnitude, that is, whether the difference has some extent and is therefore nonzero.

We can then understand cases of parity as follows: "If A and B are on a par, then their evaluative difference does not favour one alternative over the other - it has no direction - but it nevertheless has magnitude" (Chang 2016: 195). Cases of parity thus differ from cases in which A is better than B and cases in which B is better than A, since, in cases of the latter two sorts, the evaluative difference between the options is biased toward one of the options. Cases of parity also differ from cases in which $\mathrm{A}$ and $\mathrm{B}$ are equally good since in cases of the latter sort, the evaluative difference between the two options is not only not biased, but also has zero magnitude. Moreover, there is nothing in the characterization of parity under consideration that requires cases of parity to be cases of imprecise equality. In particular, the characterization does not require that the evaluative difference between the options not be large.

I will not attempt to decisively weigh in on whether Chang's controversial characterization of parity is revealing or confounding in relation to the familiar cases of parity that Chang focuses on and that fit with the idea that options that are on a par, though not rankable in relation to one another, are in the same neighborhood in terms of their overall value relative to what matters in the situation. What must be noted, given my purposes, is that the characterization gives no answer to the question that I seek to address (regarding the notion of parity that I am concerned with in this chapter and that captures the key features of the familiar cases). That question is as follows: Allowing, provisionally, that there could be options that are on a par but not roughly equally good (and so not imprecisely equally good), in what sense would such options be in the same neighborhood in terms of their overall value relative to what matters in the situation?

To answer this question, it helps to think about grading systems and how the extent to which they can be sensibly refined depends on how simple and similar the options compared are. ${ }^{12}$ If, for example, one is evaluating the books in one's office on the basis of their suitability to figure in some impromptu resistance training, and this is purely a function of their weight, one may be able to not only divide them up into broad classes that reflect "different grades or levels of quality" relative to what matters, ${ }^{13}$ such as "marginal," "good," "very good," or "excellent," but also to rank them and assign them much more precise scores based on their weight. If, however, one is evaluating books from a variety of very different disciplines for a dissertation prize, it might be misguided to suppose that, with enough time and insight, one could rank each dissertation 
in relation to every other dissertation. ${ }^{14}$ Instead, it might be that properly evaluating the dissertations involves sorting them into "grade classes," such as "marginal," "good," "very good," and "excellent," and allowing that, while options that are similar enough might be rankable in relation to each other, options that are very different might not be. Options of the latter sort might only be comparable as in the same class or as on a par. Since the grade classes are, by hypothesis, inevitably quite broad, two options in the same class can be such that one of the options is considerably better than the other. It therefore cannot be assumed that options in the same grade class qualify as roughly equally good.

Suppose, for instance, that two physics dissertations fall in the class of good books. One of the physics dissertations, P*, might be considerably better than the other, $\mathrm{P}$, just as the improved compassionate option in the previously described summer plan case is considerably better than the original compassionate option, though both options are good. Were all the dissertations quite similar to one another, it might be possible to subdivide the good dissertations into two distinct sets corresponding to two more refined evaluative classes (that do not overlap and together contain all and only the good dissertations), with one set containing the better of the two good physics dissertations, $\mathrm{P}^{*}$, and the other set containing the worse of the two good physics dissertations, $\mathrm{P}$, and the dissertations in the former set all being better than the dissertations in the latter set. This need not, however, be possible if some of the good dissertations (which might include, in addition to the two physics dissertations, a creative writing dissertation) are very different from each other and not comparable as one better than the other or as exactly equally good. If, for example, the good creative writing dissertation, C, cannot be ranked in relation to either of the two good physics dissertations, then there is no way of subdividing the good dissertations into two distinct sets with the dissertations in the set containing $\mathrm{P}^{*}$ all being better than the dissertations in the set containing P. The evaluative neighborhoods (or grade classes) that options that are on a par belong to are thus sometimes inevitably quite wide-ranging - wide-ranging enough to contain options that are not sufficiently close in value to qualify as roughly equally good, including (1) some pairs of similar options - such as $\mathrm{P} *$ and $\mathrm{P}$ in the case at hand - that are such that one of the two options is considerably better than the other, as well as (2) some pairs of options - such as $\mathrm{P}$ and $\mathrm{C}$ in the case at hand - that are not rankable in relation to each other and do not qualify as roughly equal in value because there is another option $-\mathrm{P}^{*}$ in the case at hand - that is considerably better than one of the original options but not better than the other. As such, it does not follow from the fact that options that are on a par share the same evaluative neighborhood that they are roughly equally good, even if the neighborhood cannot be subdivided into two narrower evaluative classes. 
Notably, one might consider weakening the idea of rough equality so that pairs of options - including pairs of similar options that are such that one of the options is considerably better than the other - can qualify as roughly equal if the options share a neighborhood that, due to some of the options in the neighborhood not being rankable in relation to each other, cannot be subdivided into two neighborhoods that figure as distinct grade classes (wherein all the options in one of the grade classes are better than all the options in the other grade class). But this just leaves us without a label for the less inclusive subset of cases we normally think of as cases of rough equality and papers over the real distinction between cases of parity that fit with the strict notion of rough equality and cases of parity that fit only with the weaker revisionary notion. Whatever labels we settle on, it is important to recognize that cases of parity can fail to qualify as cases of rough equality in the more standard, stricter sense of "rough equality."

Significantly, if one is receptive to the possibility of parity but skeptical about Chang's idea that, if A and B are on a par, then their evaluative difference has "magnitude" but no "bias," one can, in light of the preceding reasoning, refrain from taking Chang's idea on board and still have something illuminating to say about what it is for two options to be on a par (whether or not they are imprecisely equally good): if A and B are on a par, then, though not rankable in relation to one another, they share a class in a grading system that can be used to grade A and B and that is, in relation to this task, as refined as can be (assuming distinct, nonoverlapping grade classes). Alternatively, those sympathetic to Chang's idea might attempt to use the distinction between cases of parity that are cases of imprecise equality and cases of parity that are not cases of imprecise equality to try to illuminate talk of magnitude and (lack of) bias in cases of parity, emphasizing that cases of parity can involve options that are either more or less close in value, but that either way, reason does not favor one option over the other. Of course, strictly speaking, the assumption that $\mathrm{X}$ and $\mathrm{Y}$ are close in value does not imply that $\mathrm{X}$ and $\mathrm{Y}$ have a difference in value with some zero or nonzero magnitude, but rather that, were some third alternative, W, considerably better than one of the original options, it would also be better than the other. Still, talk of magnitude without bias might seem more promising than when first encountered.

In the end, what I really want to emphasize is that understanding parity in accordance with the preceding discussion captures the key features of (the notion of) parity (that I am here concerned with) - namely, that options that are on a par are not rankable in relation to each other, but are in the same neighborhood in terms of their overall value relative to what matters in the situation; moreover, it allows that there are instances of parity that are not instances of imprecise equality (in the sense I describe early in the chapter). The idea that options that are on a par might not be imprecisely equally good has been previously embraced; it has not, 
however, been previously explained in relation to the central idea that options that are on a par are in the same neighborhood (in terms of their overall value relative to what matters in the situation at issue). Given that options that are on a par are in the same neighborhood, it is natural to conclude that they must be close in value, and so roughly equally good. It is, I hope, illuminating to see why this need not be so.

\section{Acknowledgments}

I wish to thank Henrik Andersson, Ronald de Sousa, Anders Herlitz, Elijah Millgram, Mike White, and participants at the workshop "Incommensurability: Vagueness, Parity and Other Non-Conventional Value Relations" at the Institute for Future Studies (Stockholm, December 2019) for helpful comments on earlier drafts of this chapter. I am also grateful for supporting research funds from the Charles H. Monson Esteemed Faculty Award.

\section{Notes}

1 The quoted description of the trichotomy thesis is from Chang (2002: 661). For reasons that will be discussed later, Chang rejects the thesis.

2 The possibility of options being "imprecisely equally good" is discussed by, for example, Parfit (2016). Earlier related discussion on "rough comparability" can be found in Parfit (1984: 431). Notably, I am not using "roughly equally good" and "imprecisely equally good' as interchangeable, as is sometimes done. In particular, as I am using "roughly equally good," two options that are roughly equally good can fail to be imprecisely equally good since they might be rankable in relation to one another.

3 Although the (partial) description of options that are on a par as "in the same neighborhood" is borrowed from Chang (2016), Chang does not cast all cases of parity as cases of imprecise equality. More on this later in the chapter. Note that I will put aside, as not the conception of parity at issue in this chapter, the "permissible-attitudes" conception of parity. According to the permissible-attitudes conception, two options " $x$ and $y$ are on a par if and only if it is rationally permissible to prefer $x$ to $y$ and also rationally permissible to prefer $y$ to $x$ " (Rabinowicz 2008: 30; see, relatedly, Gert 2004 and Rabinowicz 2012). Though defensible for certain purposes (at least when suitably qualified or refined), the permissible-attitudes conception of parity allows that two options can qualify as on a par even if the choosing agent rationally permissibly strongly prefers one of them. In particular, if it is rationally permissible to prefer getting a slice of chocolate cake over getting a slice of apple pie, and it is rationally permissible to prefer getting a slice of apple pie over getting a slice of chocolate cake, then this conception of parity counts "getting a slice of chocolate cake" and "getting a slice of apple pie" as on a par, even if I am the one choosing, and I rationally permissibly strongly prefer getting a slice of chocolate cake. It thus seems unsuitable with respect to an inquiry concerning the evaluation of options relative to all that matters in the case at hand, including, in cases involving multiple rationally permissible preferences, the fit between the options and the rationally permissible preferences of the choosing agent. In any case, my interest here is in conceptions 
of parity that are tied to the familiar idea that if two options are on a par, then they are in the same neighborhood (in terms of their overall value relative to what matters in the situation); the permissible-attitudes conception of parity is not tied to this familiar idea, at least not directly enough to clearly shed light on the question that, as I will presently explain, I will be seeking to address - namely, "Could two options that are not rankable in relation to one another be in the same neighborhood (in terms of how valuable they are overall relative to what matters in the situation at hand) if they are not roughly equally good, and, if so, how could this be realized?" See, relatedly, note 5 .

4 This conclusion fits neatly with Erik Carlson's suggestion that "parity should imply a certain degree of similarity, with respect to value," and "hence, if $a$ is on a par with $b$, and $c$ is much better than $a$, then $c$ must be better than $b$, as well"; see Carlson (2010: 125) and keep in mind that, as indicated in section 3 , for two options to be close in value, it must be that were another option, W, considerably (i.e., quite a lot rather than just a little) better than one of the original options, it would also be better than the other.

5 A version of the contrast between the permissible-attitudes approach to understanding parity, discussed in note 3 , and the rough-equality approach can be found in Qizilbash (2018). I do not mean to suggest that all viable views of parity fit one or the other approach (given my description of the approaches). To the contrary, my aim is to defend a view that suggests that parity can be helpfully understood in a way that does not fit with either of these two approaches. Ruth Chang provides a characterization that also arguably occupies a different space, though it is, as will become apparent when I discuss the characterization later, one that I do not want to commit to, in part because it is not clear to me that it is defensible and in part because it does not answer the question at the end of note 3 that I will soon turn to and then attempt to answer in the rest of the chapter.

6 See Andreou, Choosing Well (unpublished manuscript). I thank the editors for inviting me to share this more complete presentation of my view.

7 See Chang (1997: section III.7) for a discussion of the small-improvement argument in the context of an introduction to philosophical issues associated with the idea of incomparability. See also, for example, de Sousa (1974) and Raz (1986: chapter 13) for earlier variations of the argument. (A particularly interesting complication related to de Sousa's variation is flagged in note 8 below.) As Johan Gustafsson and Nicolas Espinoza (2010: 754) note, "there are both preferential and axiological versions of the argument." Although Gustafsson and Espinoza put forward what might appear to be a decisive argument against the preferential version, a rebuttal to their challenge is provided in Carlson (2011). Both preferential and axiological versions of the argument continue to play a pivotal role in discussions concerning comparability.

8 Interestingly, in de Sousa's variation of this argument, wherein the agent is supposed to be torn between "keeping her virtue" and accepting a "tempt[ing]" monetary reward for "losing it" (1974: 544-545), the improvement of the monetary reward does not seem small $(\$ 1,500$ versus $\$ 1,000)$. It might be suggested that, although the difference seems large, it might qualify as small if $\$ 1,000$ will make a big difference in the agent's life but an extra $\$ 500$ on top of that will not be very significant. This complication seems like a dispensable distraction relative to de Sousa's reasoning, which only requires an option that is clearly better than $\$ 1,000$, but I find the complication quite suggestive. As will become apparent, considering more than small improvements in small-improvement type arguments is, I think, a good move to make 
in terms of helpfully complicating debate about parity, rough equality, and incomparability. According to the view I will develop, even given moderateto-large-improvement scenarios that do not enable a ranking of the options, the options may still be comparable as on a par.

9 See, relatedly, Qizilbash (2018) regarding the “'mark of parity' on the rough equality view."

10 This case, with the addition of an improved option, $A^{*}$, that I will get to shortly, is drawn from my "Incomparability and the Huge Improvement Arguments" (2021), in which the case (which I first presented at the Tanner Humanities Center in 2016) figures in an assessment of a variation of the small-improvement argument for incomparability - namely, the hugeimprovement argument for incomparability. My tentative suggestion there is that the case raises problems for the huge-improvement argument for incomparability. See, relatedly, Martijn Boot's discussion of the "large improvement phenomenon" (2017), wherein options A and B are such that A is not better than $\mathrm{B}, \mathrm{B}$ is not better than $\mathrm{A}$, and yet $\mathrm{A}$ and $\mathrm{B}$ cannot qualify as equally good, not even roughly equally good, because $A *$, which is a considerably improved version of A, is better than A but not better than B. Boot (2017) counts largeimprovement cases as cases involving incomparable options (though he uses the term "incompletely comparable" based, in part, on the idea that, although the options are "overall incomparable" - i.e., incomparable with respect to all the "relevant values taken together" - they are "usually comparable with respect to the [relevant] values [taken] separately" (316)). A notable complication that I will flag but not delve into here is that, for Boot (2017), all the evaluations in play must be "detached from...subjective preference" (317); as such, they may fail to capture the overall evaluation of the options relative to all that matters in the case at hand from the point of view of practical reason, which can include the fit between the options and the taste or preferences of the choosing agent (Andreou, 2021).

11 Notably, my reasoning later in the chapter suggests that, although, in certain cases, parity can exhibit a persistence in the face of considerable improvements that speaks against counting the options as imprecisely equally good, the persistence need not be so radical that the cases should be understood as cases of incomparability rather than cases of parity. For some interesting discussion regarding persistent unrankability, see Handfield and Rabinowicz (2018) and Herlitz (2020).

12 In this paragraph and the next, I build on my suggestion, in Andreou (2015), that "as we move away from toy cases and introduce more subtleties, the categories used in the comparison of options may involve thinner concepts with broad application. Where the most refined categories in play are extremely broad, there is a great deal of room for parity to figure as highly pervasive" (21). Notably, and as I explain in Andreou (2015), like Nien-hê Hsieh (2005), "I associate parity with the possibility of options 'clumping' together..., but my view concerning this possibility and its implications differs substantially from Hsieh's.... According to Hsieh's understanding of parity and clumpiness, parity amounts to equality" (Andreou 2015: 15, note 15).

13 The quoted phrase is borrowed from Andreou (2020b).

14 A variation of this example and of my discussion of it in the remainder of this paragraph and the next two paragraphs appears in my Choosing Well (unpublished manuscript). 


\section{References}

Andreou, C. (2015), 'Parity, Comparability, and Choice', Journal of Philosophy CXII: 5-22.

Andreou, C. (2020a), 'Empowering Rationality: Rethinking the Limits of Rational Choice’, American Philosophical Quarterly 57: 105-116.

Andreou, C. (2020b), 'In a Different League: Intransitivity, Betterness, and LeagueBased Satisficing', in A. Sauchelli (ed.), Derek Parfit's Reasons and Persons: An Introduction and Critical Inquiry (Routledge).

Andreou, C. (2021), 'Incomparability and the Huge Improvement Arguments', American Philosophical Quarterly 58/4: 307-318.

Boot, M. (2017), 'Problems of Incommensurability', Social Theory and Practice 43: 313-342.

Carlson, E. (2010), 'Parity Demystified', Theoria 76: 119-128.

Carlson, E. (2011). 'The Small-Improvement Argument Rescued', Philosophical Quarterly 61: 171-174.

Chang, R. (1997), 'Introduction', in R. Chang (ed.), Incommensurability, Incomparability, and Practical Reason (Harvard University Press).

Chang, R. (2002), 'The Possibility of Parity', Ethics CXII/4: 659-688.

Chang, R. (2016), 'Parity, Imprecise Comparability, and the Repugnant Conclusion', Theoria 82: 182-214.

de Sousa, R. (1974), 'The Good and the True', Mind 84: 534-551.

Gert, J. (2004), 'Value and Parity', Ethics 114: 492-510.

Gustafsson, J., and Espinoza, N. (2010), 'Conflicting Reasons in the SmallImprovement Argument', Philosophical Quarterly 60: 754-763.

Handfield, T., and Rabinowicz, N. (2018), 'Incommensurability and Vagueness in Spectrum Arguments', Philosophical Studies 175: 2373-2387.

Herlitz, A. (2020), 'Spectrum Arguments, Parity and Persistency', Theoria 86: 463-481.

Hsieh, N. (2005), 'Equality, Clumpiness and Incomparability', Utilitas xvii/2: 180-204.

Parfit, D. (1984), Reasons and Persons (Clarendon Press).

Parfit, D. (2016), 'Can We Avoid the Repugnant Conclusion?', Theoria 82: 110-127.

Qizilbash, M. (2018), 'On Parity and the Intuition of Neutrality', Economics and Philosophy 34: 87-108.

Rabinowicz, W. (2008), 'Value Relations', Theoria 74: 18-49.

Rabinowicz, W. (2012), 'Value Relations Revisited', Economics and Philosophy 28: 133-164.

Raz, J. (1986), The Morality of Freedom(Clarendon Press). 